\title{
EDITORIAL
}

\section{Paediatric infectious diseases in Greece: Insights from a tertiary reference unit and perspectives for the future}

\author{
MARIA THEODORIDOU
}

First Department of Paediatrics, University of Athens School of Medicine, 11527 Athens, Greece

Received January 12, 2020; Accepted April 24, 2020

DOI: $10.3892 /$ etm.2020.9418

'Life is short, art is long'

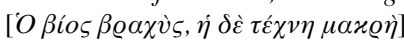

Hippocrates

The history of paediatric infectious diseases (PID) is closely connected with the specialty of paediatrics as infections comprise the major causes of childhood morbidity and mortality (1). During the past century, the paediatric population in Greece, as in all European countries, suffered from many contagious diseases, such as tuberculosis, diphtheria, malaria, poliomyelitis, meningitis and enteric infections. The difficult social situations as poverty, wars, movements of population and the clima were favorable for infectious diseases transmission.

In 1897, the 'Aghia Sophia' Children's Hospital, the biggest until today paediatric hospital in Greece, was inaugurated. From its initiation, a specialized unit was devoted for the isolation and medical treatment of children with infectious diseases. The unit had a capacity of 30 beds and the annual number of admissions exceeded the 900 (2). In 1950, the unit was named Pavilion 'MAKKA', honoring Professor George N. Makkas (1877-1946), Professor of Paediatrics at the University of Athens School of Medicine (Athens, Greece), for his fundamental contribution in modern Paediatrics and PID in Greece; Professor George N. Makkas had been professor - clinical director of the First Department of Paediatrics of the University of Athens during the periods 1929-1935 and 1936-1946 (Table I). For more than one century, the PID unit at the 'Aghia Sophia' Children's Hospital has been the reference and epidemiologic surveillance PID centre for the central, south Greece and islands (3). Data from its long lasting service represents

Correspondence to: Professor Maria Theodoridou, First Department of Paediatrics, University of Athens School of Medicine, 11527 Athens, Greece

E-mail: mpapagrig@med.uoa.gr

Key words: Paediatric infectious diseases, Greece, 'MAKKA' unit, 'Aghia Sophia' Children's Hospital, AIDS, medical education, Institute of Paediatric Virology, inauguration speech the evolution of infectious diseases over time in Greece. The patients registry was excessively used to give valuable information for epidemic outbreaks, severe complications from the diseases or vaccines to public health authorities in order the proper measures to be taken as intensification of vaccination for certain disease or change of immunization schedule. The initial period of 20th century, before the discovery of antimicrobial medicines and vaccines, was characterized from the big number of cases of tuberculosis, poliomyelitis, diphtheria, as well as cases of purulent meningitis, scarlet fever, hepatitis, typhoid fever and other enteric infections. At that time, the severe prognosis of the diseases and the transmissibility of the pathogens were important factors for the creation of social marginalization and extensive fear for the infectious diseases. The unit functioned as quarantine. It is worth noticing that the presence of the mother next to her sick child was allowed for the first time at the end of 1960's.

The following years, the discovery of sulfonamides, penicillin, anti-tuberculosis drugs, newer antibiotics and vaccines led to a remarkable change in paediatric mortality related to infectious diseases. Already from 1970, paediatric cases of tetanus, diphtheria, and poliomyelitis were rare. In 1970, due to smallpox outbreak in Yugoslav, a mass smallpox vaccination was implemented in Greece and many vaccinated against smallpox children were hospitalized at the PID unit with severe central nervous system complications. This fact made easier the decision for the interruption of smallpox vaccination. Smallpox returned in timeliness as a possible biologic threat in preparation of Olympic Games of 2004.

The reported outbreaks of infectious diseases highlighted the existing gaps of vaccination coverage in childhood. As an example in the period 1982-1983, there was an epidemic outbreak of measles (4). Although the vaccine was available 15 years earlier, studies of that period revealed low vaccination coverage with measles vaccine; the detected vaccination rate was less than 50\%. In 1987, there was also an outbreak of pertussis with 94 admissions in the unit (5). The investigation for the cause of outbreak showed again low coverage with the vaccine against pertussis due to fear for severe adverse effects. Another outbreak that guided to change of vaccination 
Table I. Professors - clinical directors of the First Department of Paediatrics of the University of Athens (Athens, Greece) since 1855 - modified by the official website of the First Department of Paediatrics of the University of Athens (17).

\begin{tabular}{ll}
\hline Period & Professor - clinical director \\
\hline $1855-1882$ & Antonios Vitsaris \\
$1879-1899$ & Anastasios Zinnis \\
$1899-1906$ & Nikolaos Pezopoulos \\
$1906-1910$ & Christos Malandrinos \\
$1910-1912$ & Nikolaos Dellaportas \\
$1912-1928$ & Christos Malandrinos \\
$1928-1929$ & Anastasios Aravantinos \\
$1929-1935$ & George Makkas \\
$1935-1936$ & Konstantinos Choremis \\
$1936-1946$ & George Makkas \\
$1946-1966$ & Konstantinos Choremis \\
$1966-1968$ & Evaggelos Danopoulos \\
$1968-1993$ & Nikolaos Matsaniotis \\
$1993-1998$ & Christos Kattamis \\
$1998-2000$ & Aikaterini Metaxotou \\
$2000-2018$ & George Chrousos \\
$2018-t 0 d a y$ & Christina Kanaka Gantenbein
\end{tabular}

schedules was the rubella outbreak in 1993 when many cases were diagnosed with congenital rubella syndrome $(6,7)$. The vaccine after this outbreak was recommended not only for girls, but also for boys and the age of implementation was changed from puberty to pre-school age.

The PID unit at the 'Aghia Sophia' Children's Hospital has been the reference centre for bacterial meningitis in Greece. Indicatively, from 1975 till 1990, 1,846 children with bacterial meningitis were admitted in the unit (8). The most frequently detected pathogens were Neisseria meningitidis (63\%), Haemophilus influenzae (16\%) and Streptococcus pneumoniae (14\%). The number of cases was practically decreased after the implementation of the Hib vaccine in 1994 and of the vaccine against Neisseria meningitides type C in 2001. The prompt diagnosis, the usage of effective antibiotics and the improved experience of health care personnel contributed to the remarkable improvement of prognosis of bacterial meningitis in children. Another frequent cause of admissions at the unit has been chickenpox causing severe complications in immune-compressed children. The tremendous progress in chemotherapy of malignant diseases over the years changed the life expectancy of the sick children; chicken pox had been a severe threat for their life before the era of antiviral treatment with acyclovir (9).

The paediatric interest for the infectious diseases decreased gradually due to progress in many fields (drugs, vaccines, improvement of socioeconomic status) till the end of 1980's, when the syndrome of acquired immunodeficiency (AIDS) first appeared. In the PID unit, the unique for Greece reference centre for paediatric AIDS was established and multi-transfused children and adolescents as well as babies born from HIV-positive mothers started to be admitted for treatment and follow up. The initial period was characterized by revival of fear of the past. The society did not accept children as victims of the disease and rejected them. The rapid evolution of anti-retroviral treatment and the achievement of prevention of vertical transmission with zidovudine from 1994 contributed in the impressive progress in the field. There is, now, no need for extension of infectious diseases units as patients have their treatment on outpatient basis; AIDS, as it is well known, became from acute a chronic infectious disease (10).

At the beginning of the 21th century due to infectious threats, such as the severe acute respiratory syndrome (SARS) in 2003, the A/H1N1 influenza outbreak in 2009 and the Middle East respiratory syndrome (MERS) in 2012, the PID unit was considered as the most appropriate place for the construction of two rooms with negative pressure; fortunately, these rooms remain for the present time - on October 2019 - still unused. In 2012, the function of the unit changed due to internal reconstruction of the main hospital and sick children with infectious diseases started to be treated in single rooms in the main hospital. Only children with HIV infection remained for their follow up in the old unit as outpatients.

All these years, the PID Unit has been a state-of-the-art centre for medical education of paediatric trainees and medical students. All junior doctors specializing paediatrics at the 'Aghia Sophia' Children's Hospital had the chance to gain valuable experience in infectious diseases attending 3 months in the unit. Moreover, the unit has been the centre for subspecialization in PID at the 'Aghia Sophia' Children's Hospital in Athens, Greece.

One might wonder what the main concerns for the infectious diseases are in the 21th century. There are many remaining challenges, such as illness that affect children in the developing world, opportunistic infections that affect immunocompromised children, emerging infections, antimicrobial resistance, new vaccines and new drugs. The genomic era of medicine and molecular biology will lead to new insights into pathogenesis, diagnosis and treatment of infections. PID will remain in the centre of scientific progress having always as a priority the improvement of children's health regardless the part of the world they live. Future PID professionals should definitely have more prevention-based tools and therapeutic choices towards infections and for this reason further research is required. Medical education should be the priority for all of us; well educated paediatric professionals in PID are vital aim, which can offer the basis for the upcoming changes in the management of PID by future medicine. The rapidly expanding field of paediatric virology is an excellent example of the necessity of upgrading the level of undergraduate, postgraduate and continuing paediatric training (11-15); this mission is expected to be fulfilled by the newly founded Institute of Paediatric Virology (16).

\section{Acknowledgements}

This article is published in the context of the foundation of the Institute of Paediatric Virology (IPV; https:// paediatricvirology.org) based on the island of Euboea (Greece), under the auspices of the World Academy of Sciences (WAS) 
and the support of the Department of Clinical Virology of the University of Crete School of Medicine and the First Department of Paediatrics of the University of Athens School of Medicine. I would like to thank all the members of the IPV for their valuable comments and corrections.

\section{Funding}

No funding was received.

\section{Availability of data and materials}

Not applicable.

\section{Authors' contributions}

MT contributed to the conception and design of this manuscript, wrote the original draft, edited and critically revised the manuscript, read and approved the final manuscript.

\section{Ethics approval and consent to participate}

Not applicable.

\section{Patient consent for publication}

Not applicable.

\section{Competing interests}

MT has been director of the PID unit at the 'Aghia Sophia' Children's Hospital (Pavilion 'MAKKA'). MT is Co-founder of the Institute of Paediatric Virology (IPV).

\section{References}

1. Shulman ST: The history of Pediatric Infectious Diseases. Pediatr Res 55: 163-176, 2004.

2. Zoumboulakis D: Pavilion of Infectious Diseases (MAKKA) 1897-1974. Ann Clin Paediatr Univ Atheniensis 21: 173-179, 1974.
3. Theodoridou M, Gelesme A, Lourida A and Zoumboulakis D: Aghia Sophia Children's Hospital, Department of Infectious Diseases. Hospital admissions: 1975-1990. Arch Hellen Med 9: 194-197, 1992.

4. Maragos C, Adam H, Theodoridou M, Manolaki N, Georgakis A and Zoumboulakis D: Effect of a measles epidemic on immunization. Arch Hellen Med 2: 286, 1985

5. Vazeou A,Theodoridou MA and Zoumboulakis D: Pertussis 1987: Facts and problems. Paediatriki 53: 169-173, 1990.

6. Theodoridou M, Agrafioti C, Sotiropoulou F, Liapi G, Mostrou G and Zoumboulakis D: Acute rubella encephalitis (Report of nine cases). Ann Clin Paediatr Univ Atheniensis 42: 181-183, 1995.

7. Panagiotopoulos T, Antoniadou I, Valassi-Adam E and Berger A: Increase in congenital rubella occurrence after immunisation in Greece: Retrospective survey and systematic review. BMJ 319: 1462-1467, 1999.

8. Theodoridou MN, Vasilopoulou VA, Atsali EE, Pangalis AM, Mostrou GJ, Syriopoulou VP and Hadjichristodoulou CS: Meningitis registry of hospitalized cases in children: Epidemiological patterns of acute bacterial meningitis throughout a 32-year period. BMC Infect Dis 7: 101, 2007.

9. Theodoridou M,Laina I, Hadjichristodoulou C and Syriopoulou V: Varicella-related complications and hospitalisations in a tertiary pediatric medical center before vaccine introduction. Eur J Pediatr 165: 273-274, 2006.

10. Papagrigoriou-Theodoridou M: Paediatric HIV Infection. Pediatriki 60: 173-176, 1997.

11. Mammas IN, Greenough A, Theodoridou M and Spandidos DA: Paediatric Virology: A new paediatric subspecialty? A proposal at the Workshop on Paediatric Virology, Athens, October 10, 2015. Exp Ther Med 11: 3-5, 2016.

12. Mammas IN and Spandidos DA: The subspecialty of Paediatric Virology: A 'mosaic tile' in future Paediatrics. Exp Ther Med 12: 539-540, 2016.

13. Mammas IN, Theodoridou $M$ and Spandidos DA: The development of the Paediatric Virology Study Group: Ten years in the making. Exp Ther Med 13: 363, 2017.

14. Mammas IN, Greenough A, Theodoridou M and Spandidos DA: Does Europe need paediatric virologists? Exp Ther Med 16: 2783-2784, 2018

15. Mammas IN, Greenough A, Theodoridou M and Spandidos DA: Educational pathways in Paediatric Virology: Pros and cons. Exp Ther Med 18: 3260-3262, 2019.

16. Mammas IN, Greenough A, Theodoridou M and Spandidos DA: The foundation of the Institute of Paediatric Virology on the island of Euboea, Greece (Review). Exp Ther Med (In Press).

17. First Department of Paediatrics of the University of Athens: Aim - History, 2020 (In Greek). https://www.firstpediatrics-uoa. gr/stoxoi-storia/.

This work is licensed under a Creative Commons Attribution-NonCommercial-NoDerivatives 4.0 International (CC BY-NC-ND 4.0) License. 